\title{
Inhibition of SARS-CoV-2 Replication by Acidizing and RNA Lyase-Modified Carbon Nanotubes Combined with Photodynamic Thermal Effect
}

\author{
Jianshe Yang* \\ Central Laboratory of $3^{\text {rd }}$ Hospital, the Chinese University of Hong Kong (Shenzhen), Shenzhen, China
}

\begin{abstract}
The first patient suffering from severe acute respiratory syndrome (SARS) was identified in December 2019 in Wuhan, China. Physicians and scientists consequently diagnosed and identified this case of SARS as COVID-19, which was caused by infection with SARS-CoV-2, a new coronavirus. To date, it has spread as a global pandemic, with more than 2.5 million confirmed patients and 175 thousand deaths. Unfortunately, we have yet to find a specific effective therapy; although, some maintenance therapies are known to improve symptoms, partially referencing the experiences from anti-SARS-CoV and the Middle East respiratory syndrome. In addition, many clinical trials are completed or ongoing. Accordingly, a new strategy for development of therapeutic drugs is urgently needed. Here, we propose to prepare a kind of carbon nanotube with functions to exert acidification for cytoplasmic and local cellular temperature-rising through photothermal conversion, according to the physical and chemical nature of carbon nanotubes having been well applied to facilitate such a response. Dexterously, we will put the above effects into practice to inhibit SARS-CoV-2 replication with respect to the biological nature of coronavirus.
\end{abstract}

\section{Introduction}

Coronavirus $(\mathrm{CoV})$ is an enveloped, single-stranded, positivestranded RNA virus and widely distributed among humans, other mammals, and birds. It can cause respiratory, intestinal, liver, and nervous system diseases. Seven species of CoVs are currently known (including the newly identified severe acute respiratory syndrome coronavirus 2 (SARS-CoV-2) which can make humans sick. Among these, four viruses-229E, OC43, NL63 and HPU1-are ubiquitous and usually cause common cold symptoms in immunecapable individuals. The other three species-SARS-CoV, Middle East respiratory syndrome (MERS)-CoV, and SARS-CoV-2-have their origins in zoonotic diseases and can cause fatal diseases in humans. SARS-CoV is the pathogen that caused SARS outbreaks in Guangdong, China during 2002 and 2003, while MERS-CoV is the pathogen that caused the outbreak of the severe respirato-

Keywords: SARS-CoV-2; COVID-19; Inhibition; Carbon nanotube; Acidize; RNA lyase; Photodynamic thermal effect.

Abbreviations: SARS, severe acute respiratory syndrome; SARS-CoV-2, severe acute respiratory syndrome coronavirus-2; COVID-19, coronavirus disease 2019; 2019-nCoV, 2019 novel coronavirus; CNT, carbon nanotube; MWCNT, multi-walled carbon nanotube; p-WCNT, primary multi-walled carbon nanotube; f-WCNT, functional multi-walled carbon nanotube; ACE2, angiotensin-converting enzyme 2 . Received: March 27, 2020; Revised: April 15, 2020; Accepted: April 23, 2020

${ }^{*}$ Correspondence to: Jianshe Yang, Central Laboratory of $3^{\text {rd }}$ Hospital, the Chinese University of Hong Kong (Shenzhen), Shenzhen, 518172, China. Tel: +86-75528932833-8756, E-mail: yangjs@impcas.ac.cn

How to cite this article: Yang J. Inhibition of SARS-CoV-2 Replication by Acidizing and RNA Lyase-Modified Carbon Nanotubes Combined with Photodynamic Thermal Effect. J Explor Res Pharmacol 2020;5(2):18-23. doi: 10.14218/JERP.2020.00005. ry disease in the Middle East in 2012. The high prevalence and widespread distribution of $\mathrm{CoV}$, its genetic diversity and frequent recombination, and the increasing human-animal interactions as well as frequent cross-species infections and occasional spills, can make CoVs occur periodically in humans, leading to outbreaks of specific epidemics. $^{1-4}$

From late December 2019, many cases of viral pneumonia were found in Wuhan City, Hubei Province, China. Based on the virus-typing test, a new $\mathrm{CoV}$ was detected in the laboratory on January 7, 2020, and the pathogenic nucleic acid sequencing was completed on January 10. On January 12, the World Health Organization officially named the new $\mathrm{CoV}$ that was causing the Wuhan pneumonia epidemic as the "2019 new coronavirus (2019$\mathrm{nCoV})$ ", and on the same day, it released guidelines for clinical management. ${ }^{5-8}$

As of January 27, confirmed coronavirus disease 2019 (COVID-19) cases and suspected cases have been reported in other provinces and municipalities in China, including Hong Kong, Macao and Taiwan regions, and with the exception of Tibet; some critically patients have died. In Hubei Province, especially Wuhan City, the epidemic situation is the most serious, and the epidemic situation in Guangdong Province and Shenzhen is relatively serious. $5,7,8$

Due to the rapid development of molecular biological diagnostic technologies, such as ribonucleic acid detection, and the large amount of clinical experience accumulated in response to the SARS-CoV outbreak in 2003, physicians and scientific researchers have been able to analyze SARS-CoV-2 in a very short period of time. Hereafter, the genome-wide information of this novel virus can be used to promote accurate diagnose for viral pneumonia and to implement different degrees of intervention based on the clinical 
symptoms associated with the disease. However, due to the specificity of the pathogen, there is currently no specific effective drugs to control this type of virus and the diseases it causes. The research for developing new rapid and high-sensitivity diagnostic methods and designing or finding drugs with good anti-CoV potency will be long-term and arduous tasks.

CoVs have special physical and chemical properties. They are not acid-resistant nor insensitive to heat and will be extremely inactivated in a strongly acidic environment or at $56{ }^{\circ} \mathrm{C}$ for $30 \mathrm{~m} .^{1,2}$ Furthermore, $\mathrm{CoV}$ is sensitive to restrictive nucleic acid lyase. ${ }^{9}$ Carbon nanotubes (CNTs) are new materials that have been widely studied and used in the field of applied biology in recent years. They have small size dimensions $(10-100 \mathrm{~nm})$, high dimensionality (2D, 3D), and good light-heat conversion efficiency. Physical and chemical characteristics, such as great storage space in the inner tube and surface load capacity, will result in some excellent biological properties, such as good biocompatibility, high permeability of biological barriers, high bioabsorption rate, multi-energy surface/tube chemical functional group capability, and targeted biomolecule modification potency, etc. These characteristics provide new ideas for the development of new anti-CoV drugs. Based on this, we propose a hypothesis/project to acidify functionally CNTs to inhibit SARS-CoV-2, in order to provide theoretical support to new methods and new strategies for the prevention and control of SARS-CoV-2.

The proposal of this project has the following preferential objective circumstances: Shenzhen is currently facing the grim situation of the SARS-CoV-2 epidemic, and as of now there are no effective vaccines nor drugs to deal with and control the outbreak clinically. Due to the high-frequency mutation rate of the virus, there has been a lag in development of the vaccine and drug screening. The project we propose focuses on the physical and chemical properties of $\mathrm{CoV}$, combined with the theoretical and application research of carbon nano-functionalized materials in the field of biomedicine, to build a targetable acidification potential. The established CNT functionalized complex will interact with type II alveolar epithelial cells (the main host of SARS-CoV-2 infection), provide the body with double protection from SARS-CoV-2, and establish an interception system for SARS-CoV-2. The successful implementation of this novel therapy will provide vital support for control of the SARS-CoV-2 outbreak and will provide very important economic and social benefits.

\section{Technology development trends and status at home and abroad}

The CoVs have an irregular shape, with a diameter of about $60-220 \mathrm{~nm}$. The viral particles are enveloped by a lipid membrane. There are three types of glycoproteins on the surface of the membrane: spike protein $(\mathrm{S}$; which is a receptor binding site, cytolysis, and main antigen Loci); small envelope glycoprotein (E); and membrane glycoproteins (M; responsible for transmembrane transport of nutrients, budding and release of newborn viruses, and formation of viral envelopes). A few species also have hemagglutinin glycoprotein (HE, also known as hemagglutinin-esterase). CoV's nucleic acid sequence is non-segmented, single-stranded, positive-strand RNA, 27-31 kb in length, which represents the longest RNA virus. This positive-strand RNA has important structural characteristics; that is, the $5^{\prime}$ end of the RNA strand has a methylated "cap" and the 3 ' end has a polyA "tail" structure. This structure is very similar to eukaryotic mRNA, so that the viral genome RNA itself can play an important role in the translation template, omitting the need for the routine tran- scription process of RNA-DNA-RNA. The recombination rate between RNA and RNA of CoV is very high, which leads to a high level mutation rate. After recombination, the RNA sequence has changed, as well as the amino acid sequence, making further changes in the encoded proteins and ultimately making antigenicity changes; as such, the original vaccine and immunization will become failures. ${ }^{2}$

The viral RNA polymerase required for RNA virus replication is not present in the CoV mature particles. After it enters the host cell, the viral genome RNA is directly used as the translation template to express the viral polymerase, this enzyme is then used to complete the transcriptional synthesis of negative chain subgenomic RNA, the synthesis of various structural protein mRNA, and the replication of viral genomic RNA. mRNA synthesis of the mature various structural proteins of $\mathrm{CoV}$ occurs without post-transcriptional modification of the cutting process but directly through the RNA of polymerase and some transcription factors; this process involves a "discontinuous transcription" mechanism that identifies specific domains and performs selective one-time transcription from the negative chain RNA to form the full components of a mature mRNA. After replication, the RNA structural proteins and genomes are completed, and new $\mathrm{CoV}$ particles are assembled in the endoplasmic reticulum of host cells and secreted through the Golgi apparatus to complete their life cycle. ${ }^{2}$

Due to the above-described biological processes and physichemical characteristics, the proliferative ability of $\mathrm{CoV}$ is extremely strong and its genome and nucleic acid sequence mutation frequencies are also very high. Therefore, developing a comprehensive treatment of respiratory diseases caused by $\mathrm{CoV}$ faces huge obstacles. Though we have a variety of treatment modes for $\mathrm{CoV}$ currently, their clinical efficacies are extremely limited; in addition, the screening and development of $\mathrm{CoV}$ drugs have often lagged behind the mutation of the virus itself. Studies have confirmed that alpha-interferon and gamma-interferon are compatible as anti-SARS-CoV agents in vitro. ${ }^{10}$ Carbajo-Lozoya et al. ${ }^{11}$ found that low-concentration, non-cytotoxic tacrolimus (FK506) can strongly inhibit the growth of SARS-CoV in cultured cells, providing value for clinical treatment of SARS-CoV infection. MERS$\mathrm{CoV}$ is the second $\mathrm{CoV}$ that is highly pathogenic to humans. Studies have found that the combined use of alpha-interferon- $2 b$ and ribavirin can effectively reduce virus replication, regulate host response, and improve clinical prognosis in MERS-CoV-infected rhesus macaques. ${ }^{9}$ In another study of anti-MERS-CoV activity with known antiviral drugs, it was found that only mycophenolic acid has a lower half-effective concentration and high selectivity index, while ribavirin and interferon have anti-MERS-CoV in vitro activity and may be considered applicable to the experimental treatment studies of MERS. ${ }^{12}$

It can be seen that the current treatment of $\mathrm{CoV}$ is mainly dependent on the discovery of protease inhibitors with high specificity or as a paradigm to find more lead compounds. The heterologous recombinant expression and purification of SARS CoV protease (SARS-CoV Mpro) and the screening of positive compounds have laid the foundation for the study of SARS-CoV protease inhibitors and provided the leading compounds for the development of antiviral drugs. ${ }^{13}$

During the outbreak of SARS-CoV and SARS-CoV-2, Chinese medicine has also played an important role in symptomatic treatment but the mechanism is still unclear. Although the drugs developed based on the above paradigm have a good inhibitory effect on $\mathrm{CoV}$, because of the high frequency mutation of the genome and nucleic acid sequence of the virus, the development of vaccines and drugs is lagging behind and the spread of the epidemic is extremely unlimited due to the loss of immune efficacy against 
the mutated virus after the development of the drug. The prevention and control of $\mathrm{CoV}$ are usually summarized as follows: (1) At the policy level, the relevant departments of the state and government should introduce policies to educate people to change their bad eating habits, to not eat wild animals, and make policies to restrain them. At the same time, they should increase their investment and carry out long-term and systematic projects on viruses and pathogens, with a view towards controlling the spread of viruses, fundamentally; (2) Interception should be carried out with drug intervention before or after infection with the virus/suspected virus; (3) Passive treatment after diagnosis.

Since Iijima et al. ${ }^{14,15}$ first reported the synthetic production of CNTs in 1991, a lot of breakthrough achievements in physical, chemical, material, biological and other fields were reached in just over two decades. As of now, the dominant applications of carbon nanomaterials in the field of biomedicine are mainly manifested in the following three aspects: (1) by chemical modification of CNTs' surface and inner tube, so that functional groups or special molecules with special chemical properties are introduced to make the modified complex exhibit new chemical properties, such as strong acidity; (2) by grafting biologically-targeted molecules (i.e. sugar, glycolipid, protein, RNA, DNA, etc.) on the surface of $\mathrm{CNTs}^{16}$ to make them active towards specific organs or tissues; (3) by exploiting CNTs' superior near-infrared light absorption characteristics and good photothermal conversion efficiency to make the local region that CNTs aggregate experience a sharp rise in temperature, to more than $51{ }^{\circ} \mathrm{C}$, in order to realize photodynamic hyperthermia. ${ }^{17,18}$ Now, intelligent nano-complexes with targeted effects have been developed, which have a good application prospect in medicine and biology.

From the second level, this proposed project uses the physical and chemical properties of $\mathrm{CoV}$, and uses functionalized CNT complexes to intercept the SARS-CoV-2, to block its replication and proliferation in the body, so as to efficiently fight COVID-19.

Proposed project to acidify functionality of CNTs to inhibit SARS-CoV-2

The research content of the proposed project mainly includes the following 10 steps:

\section{Acidification of CNTS}

Using a strong acid and alkali mixing chemical process, as described in the previous reports, ${ }^{19,20}$ the original CNTs were modified into uniform 50-nm diameter primary CNTs with carboxyl and hydroxyl groups on their surface. The process first involved the original multi-walled CNTs (purchased from Shenzhen Nanoport $^{\circledR}$, China) being used to remove the metal catalyst residues in hydrochloric acid, followed by full reaction in a strong acid solution of concentrated sulfuric acid and nitric acid to obtain a homogeneous suspension. Then, by polytetrafluoroethylene membrane filtering and treatment with concentrated sulfuric acid and hydrogen peroxide solution, CNTs were finally obtained by ammonia oxidation with persulfate. The resultant CNTs were named as pWCNTs, for 'primary CNTs'.

The $\mathrm{p}$-WCNTs are analyzed by scanning electron microscope, thermogravimetric loss, Zeta potential, x-ray photoelectron spectroscopy and other equipment and methods to characterize relevant physical and chemical parameters. Preparation conditions will be optimized by calibrating the parameters, and finally to obtain high-yield, stable-surface, $\mathrm{COOH}^{-}, \mathrm{OH}^{-}$enriched p-CNTs. Subsequently, a study of $\mathrm{p}$-CNTs' regulation of $\mathrm{pH}$ values (internal and external to cells) will be carried out.

\section{Modification of p-CNTs by grafting the lung epithelial cells ACE2 ligand and SARS-CoV-2 RNA lyase}

Angiotensin-converting enzyme 2 (ACE2) is the main receptor for SARS-CoV and it has also been confirmed to have specific affinity for the S-protein of SARS-CoV-2. ${ }^{5,21}$ Studies $^{7,22}$ have shown that approximately $0.64 \%$ of human lung cells will express ACE2, and the expression on type II alveolar epithelial cells accounts for $83 \%$. Other types of cells rarely express ACE2, such as type I alveolar epithelial cells, airway epithelial cells, fibroblasts, endothelial cells and macrophages. ACE2 specific affinity ligand molecules (i.e. the receptor binding domain of ACE2, which binds S proteins of SARS-CoV-2) have been modified onto the surface of CNTs by chemical self-assembly and covalent binding. Alpha-interferon produces an effect of $\mathrm{CoV}$ RNA lyase; under the stimulation of alpha-interferon, infected cells will specifically synthesize and secrete a protein that inhibits viral protein synthetase, showing an RNA lyase-like effect and thereby blocking the replication of the virus in infected cells; such will be stored in the nanotube cavity by physical adsorption, representing the functionalized CNTs, named as f-WCNTs.

\section{Selective biological behavior of $f$-WCNTs in a multi-cell co- culture system}

Because the final objective of this project is to administer f-WCNTs in the form of a throat spray, consideration must be made for the oral mucosa and pharyngeal mucosa being contacted during the process of the drug entering the alveoli. In order to confirm whether f-WCNTs are effective on type II alveoli epithelial cells via specific affinities, the following study was designed. Different types of cells, including alveolar type II epithelial cells, were seeded on separate culture plates to obtain stable and proliferating cell clones in a limited medium. Then, the medium was changed so that it does not cross the division on the culture plate and to achieve the same system culture of all cells. Next, f-WCNTs were added evenly to the medium to observe the aggregation and phagocytosis of the f-WCNTs by different cells. It is necessary now to obtain image data of the interaction between f-WCNTs and the alveolar epithelial cells at different time points and to use scanning electron microscopy to characterize and confirm the effect of phagocytosis.

\section{4. f-WCNTs targeted transport and phagocytosis in lung}

CNTs have good active properties that facilitate penetration of the cell membrane. After modification with an ACE2 receptor-specific affinity ligand, f-WCNTs will aggregate toward type II alveolar epithelial cells with high specificity, making it easier to achieve entry into alveolar epithelial cells. Due to the surface of CNTs being fabricated with a large number of carboxyl and hydroxyl groups, the local environment inside and outside the cell can be highly acidified, which will cause cell membrane receptors and nuclear substances to undergo conformation changes, thereby inhibiting viral RNA replication through specific pathways. Meanwhile, the SARS-CoV-2-specific RNA lyase enzymes bound to the surface or adsorbed in the lumen (near-infrared light irradiation can ef- 


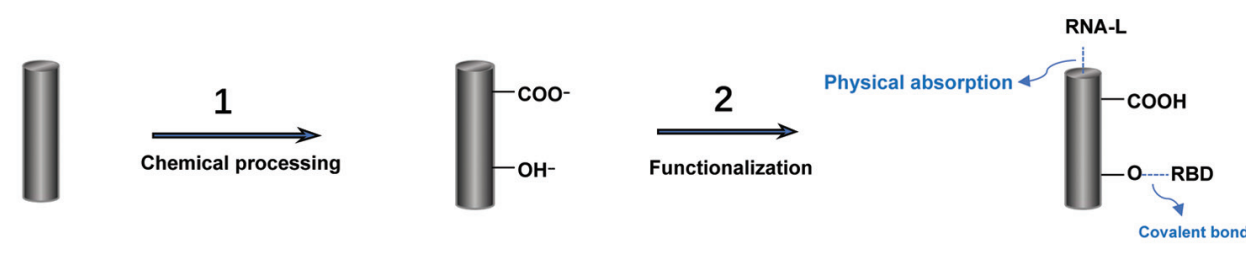

MWCNT

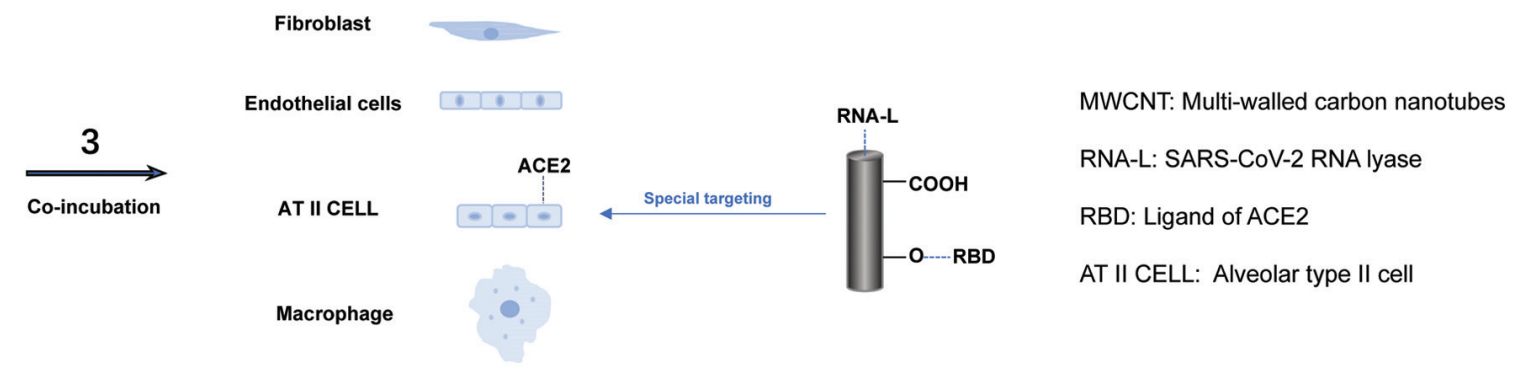

Fig. 1. Flow chart of the proposed research content steps 1-4. This flow chart described briefly the MWCNT was fabricated with strong acid and base conditional mixture in order to achieve the p-WCNT (chemical process); then modified with RNA layse and receptor binding domain (RBD) by covalent conjugation and physical absorption to get f-WCNT (functionalization); thereafter, f-WCNT was used in the multi-cell culture system interacting with SARS-CoV-2 to identify the special affinity of f-WCNT to ACE2 labeled alveolar type II cells and the inhibition capacity to SARS-CoV-2.

fectively promote CNTs to release the surface-modified molecules and alpha-interferon that is adsorbed in the lumen) will interact with the CoV RNA (Fig. 1).

Ligand receptor binding reactions enable f-WCNTs targeting to be enriched towards alveolar epithelial cells. Through this, this study focused on f-WCNTs' dynamic processes of targeted aggregation, phagocytosis and other behaviors in type II alveolar epithelial cells, with other types of cells as controls.

Description of photodynamic thermal effect of near-infrared light in steps 5 to 9

Multi-walled carbon nanotubes (MWCNTs) have high photothermal conversion efficiency in both the first biological window $(700-950 \mathrm{~nm})$ and the second biological window $(1,000-1,350$ $\mathrm{nm})$, regardless of the influence of wavelength. Previous studies $^{17,18}$ have found that whether the light of the first window (with a wavelength of $808 \mathrm{~nm}$ ) or the second window (with a wavelength of $1,090 \mathrm{~nm}$ ) as well as the interval region light (with a wavelength of $980 \mathrm{~nm}$ ) is used as the excitation source for photothermal therapy, the photothermal conversion efficiency was close to $50 \%$ when the MWCNTs, with a diameter of $10 \mathrm{~nm}$, a length of $1.5 \mu \mathrm{m}$ was irradiated. At MWCNTs' concentration of $0.1 \mathrm{mg} / \mathrm{mL}$ with irradiation under a laser using a wavelength of $1,064 \mathrm{~nm}$ and intensity of $3 \mathrm{~W} / \mathrm{cm}^{2}$ for $30 \mathrm{~s}$, the local temperature around the MWCNTs can rise from $23{ }^{\circ} \mathrm{C}$ to about $51{ }^{\circ} \mathrm{C}$, promptly.

The implementation of near-infrared laser irradiation could create a local high temperature environment of more than $50^{\circ} \mathrm{C}$ in the f-WCNTs-enriched type II alveolar epithelial cells, effectively inactivating SARS-CoV-2. This treatment will cooperate with and enhance the effect resulting from the strong acid environment and alpha-interferon, inhibiting $\mathrm{CoV}$ and achieving a triple strike to the $\mathrm{CoV}$ pathogen.

\section{Virus intrusion and blocking experiments based on pseudovi- rus}

The $\mathrm{CoV} \mathrm{S} / \mathrm{E} / \mathrm{M}$ protein expression plasmid, lentivirus packaging plasmid $(\Delta 9.2) \mathrm{m}$ and lentivirus empty vector were co-transfected into $293 \mathrm{~T}$ cells by transfection reagent; a lentiviral vector containing green fluorescent protein or luciferase reporter gene is recommended for transfection, which is convenient for determination of virus titer. Culture medium was collected at $72 \mathrm{~h}$ after transfection, and the floating cells were removed by centrifugation and filtration $(0.45 \mu \mathrm{m}$ filter membrane) to ensure the complete removal of the cells. The filtered supernatant was stored at $80^{\circ} \mathrm{C}$. A small amount of supernatant was taken for gradient dilution to infect $293 \mathrm{~T}$ cells stably-expressing receptor protein ACE2 to determine the titer of the pseudovirus. The neutralization titer of f-WCNTs was evaluated by measuring the f-WCNTs' ability to inhibit pseudovirus infection by adding equivalent amounts of pseudovirus and gradient-diluted f-WCNTs to the stably-expressing pseudovirus 293T's cell medium.

\section{Determination of the inhibitory effect of $f-W C N T s$ on the repli- cation of SARS-CoV-2 in type II lung epithelial cells}

The latest studies ${ }^{7,21}$ have described that SARS-CoV-2, like SARS$\mathrm{CoV}$, belongs to the $\beta-\mathrm{CoV}$ type, having about $70 \%$ genome sequence similarity and the same receptor (ACE2). So, they are presumed to have similar viral replication characteristics. This study pre-referenced SARS-CoV infection and its detection means, with high infection complex number of SARS-CoV-2-infected type II of lung epithelial cells. At $6 \mathrm{~h}$ after infection, different concentrations of $\mathrm{f}$-WCNTs were added to the medium to co-culture. Then, the culture medium was taken for use as the detection sample at $12 \mathrm{~h}, 24 \mathrm{~h}, 48 \mathrm{~h}$ and $72 \mathrm{~h}$ after virus infection. The titer of virus in different samples was detected by virus plaque experiments with 
VeroE6 cells, so as to determine the inhibitory effect of different concentrations of $\mathrm{f}-\mathrm{WCNTs}$ on virus replication. At the same time, the cells at each time point were collected for counting, to determine the protective effect of f-WCNTs on infected cells.

\section{Detection of $S / E / M$ protein expressions after interaction be- tween $f$-WCNTs and SARS-CoV-2-infected type II lung epithelial cells}

Mature $\mathrm{CoV}$ particles have envelope structures and at least three viral structural proteins: S, E, and M. Among them, the S protein is involved in the recognition and binding of the surface receptors of host cells and mediation of the fusion of virus $\mathrm{E}$ and the host cell membrane, which is an important antigen for immune recognition. The E protein is involved in the assembly and release of virus particles. The M protein represents the most abundant structural proteins in virus particles, which can promote membrane bending and can bind to the nuclear capsid. ${ }^{1-3}$ By using the western blotting method, the expression of these three proteins in infected cells at different concentrations of f-WCNTs at different times can be detected, providing evidence by which to judge virus viability. The structures of $\mathrm{S} / \mathrm{E} / \mathrm{M}$ proteins were characterized by freeze-drying application in electron microscopy; the binding force of $\mathrm{f}-\mathrm{WCNT}$ to $\mathrm{S}$ protein was calculated, and the conformational characteristic mechanism of inhibiting SARS-CoV-2 was simulated and analyzed.

\section{Sequence analysis and viability identification of viral RNA}

Here are two situations: (1) After the virus capsid is cleaved, if the RNA is incomplete, then identify its toxicity and viability; or (2) If the virus is intact, determine how the RNA lyases' bound to f-WCNTs work.

$\mathrm{CoV}$ is a kind of single-strand, positive-chain RNA virus. The virus genome RNA acts as an mRNA after entering the host cells, expressing the main unstructured protein and forming the RNA transcription-replication complex; subsequently, the virus genome RNA uses this complex to complete the replication of the negativechain of the genome, the transcription of the various structural protein mRNAs, and the replication of the virus genome. The integrity of the RNA chain and the high conservation of the cap structure at the $5^{\prime}$ end and the polyA structure at the $3^{\prime}$ end of the RNA chain are the guarantee that the virus can replicate and translate $\mathrm{S}$ protein normally. ${ }^{23}$ With the intervention of a strong acid environment and RNA lyase produced by $\mathrm{f}-\mathrm{WCNTs}$, the $\mathrm{CoV}$ infecting cells face a double blow, which can cause the destruction and cleavage of RNA virus capsid structure and of the transcription-replication complex. Exposed RNA may also suffer structural changes due to the action of lyase, such as breaking into different size fragments. And, of course, there is the possibility that the allosteric RNA will cause new pathogenic problems, which need to be discussed separately. These types of structural damage may lead to inactivation of the virus or reduced virulence, which can inhibit the virus. Ribonucleic acid sequencing (i.e. RNA sequencing) is used here to identify changes in viral RNA after f-WCNT intervention.

\section{Viral metabolism research in hACE2 transgenic mice}

The hACE2 transgenic mice were treated with f-WCNTs via spray; then, tissue sections were collected to observe the changes of lung tissue morphology and to verify the safety of the drugs. Moreover, SARS-CoV-2-infected hACE2 transgenic mice were treated with
f-WCNT intervention, and pathological sections were then made to detect the infiltration level and viral load of inflammatory cells in the interstitial bronchi of mice and to evaluate the efficacy of drugs. After the biological behavior of capsid toxic protein and virus RNA was systematically studied by using hACE2 transgenic mice as a virus-sensitive model, through laryngeal spray inhalation of f-WCNTs and with different concentrations and time points as variables. The effects of the different kinds of cells present in the lungs of mice after f-WCNT intervention were systematically studied, combined with the detection of respiratory-, urinary- and blood-related indices to analyze and evaluate the comprehensive effect of $\mathrm{f}-\mathrm{WCNTs}$ against SARS-CoV-2.

\section{Based on the above research, $f$-WCNT spray is prepared for prevention and treatment}

The prepared f-WCNTs was mixed with distilled water according to the volume ratio of $1: 1.8-1: 2.2$, adding appropriate amount of menthol and other edible aromatic agents, and then filling the mouth spray bottle after ultraviolet sterilization.

\section{Technical innovations and difficulties}

The technological innovation of this project lies in making full use of the physical and chemical properties of $\mathrm{CoV}$ without acid resistance and heat sensitivity, and is achieved by constructing CNT composites with targeted function exploiting acidization of the host cell's inside and outside environments. This project also allows for attacking of $\mathrm{CoV}$ from three levels, namely acidizing the cell environment, creating a photodynamic heat effect by nearinfrared laser irradiation f-WCNTs, and targeting delivery of an RNA lyase destruction virus to effectively block virus infection and replication pathways in the host cell and realizing a new strategy of prevention and treatment of CoV. Meanwhile, the technical difficulties of this project include: (1) grafting modification of receptor ACE2-specific ligands on the WCNT surface, (2) modifying and protecting the SARS-CoV-specific nuclease on the WCNTs, and (3) constructing a pseudovirus.

\section{Future directions}

Considering the ineffectiveness of existing vaccines and drugs, which have never been confirmed to be useful for SARS-CoV or MERS-CoV, we should give much more attention to specific treatments for SARS-CoV-2. We should also strengthen our focus on exploiting the physical and chemical properties of the virus to design corresponding materials to boost effective suppression of the virus, rather than stubbornly fantasizing that only molecular biological research can solve the problem of virus replication and epidemic. In a word, we should be sober, working towards a basic, simple, not necessarily ineffective treatment.

\section{Conclusions}

Strictly according to the nature of $\mathrm{CoVs}$, as they are sensitive to acidic environment and high temperature (above $56{ }^{\circ} \mathrm{C}$ lasting more than $30 \mathrm{~m}$ ), CNTs, fabricated with acidization and conjugation with special RNA lyase to exploit the capacity of photothermal conversion effect, may prove a good priority in the search for 
a means by which to inhibit SARS-CoV-2 and illuminate a whole new way for fighting COVID-19.

\section{Acknowledgments}

The author expresses his greatest thanks to the following scientists for supporting this project: Prof. Dr. Zhikang Qian of the Shanghai Pasteur Institute, Chinese Academy of Sciences, and Prof. Dr. Zhiyong Li of Lanzhou Institute of Veterinary Medicine, Chinese Academy of Agricultural Sciences.

\section{Funding}

This study was not funded by any companies or institutes.

\section{Conflict of interest}

The author declares that he has no conflict of interest.

\section{References}

[1] Weiss SR, Navas-Martin S. Coronavirus pathogenesis the emerging pathogen severe acute respiratory syndrome coronavirus. Microbiol Mol Biol Rev 2005;69(4):635-664. doi:10.1128/MMBR.69.4.635664.2005.

[2] Liu KZ. Human viral diseases (in Chinese). People's Medical Publishing House, 2010:643-665.

[3] Nikolaeva SV, Zvereva ZA, Kanner EV, Yatsyshina SB, Usenko DV, Gorelov AV. A clinical-laboratory characteristic of coronavirus infection in children (in Russian). Infectious Diseases 2018;16(1):35-39. doi:10.20953/1729-9225-2018-1-35-39.

[4] de Haan CA, van Genne L, Stoop JN, Volders H, Rottier PJ. Coronaviruses as vectors: position dependence of foreign gene expression. J Virol 2003;77(21):11312-11323. doi:10.1128/jvi.77.21.11312-11323. 2003.

[5] Zhu N, Zhang D, Wang W, Li X, Yang B, Song J, et al. A novel coronavirus from patients with pneumonia in China, 2019. N Engl J Med 2020;382(8):727-733. doi:10.1056/NEJMoa2001017.

[6] WHO. Novel coronavirus (2019-nCoV). Available from: https://www. who.int/docs/default-source/coronaviruse/situation-reports/ 20200125-sitrep-5-2019-ncov.pdf. Accessed January 25, 2020.

[7] CormanV, BleickerT,BrüninkS, DrostenC. Diagnosticdetection ofWuhan coronavirus 2019 by real-time RT-PCR. Available from: https://www. who.int/docs/default-source/coronaviruse/wuhan-virus-assayv1991527e5122341d99287a1b17c111902.pdf?sfvrsn=d381fc88_2. Accessed January 13, 2020.

[8] Huang C, Wang Y, Li X, Ren L, Zhao J, Hu Y, et al. Clinical features of pa- tients infected with 2019 novel coronavirus in Wuhan, China. Lancet 2020;395(10223):497-506. doi:10.1016/S0140-6736(20)30183-5.

[9] Falzarano D, de Wit E, Rasmussen AL, Feldmann F, Okumura A, Scott DP, et al. Treatment with interferon-a2b and ribavirin improves outcome in MERS-CoV-infected rhesus macaques. Nat Med 2013;19(10):1313-1317. doi:10.1038/nm.3362.

[10] Ji XG, Zhao YH, Zhang M, Zhao JH, Wang JY. The experimental study of $\alpha$-interferon and $\gamma$-interferon and their combined anti-SARS-CoV effect in vitro (in Chinese). Pharmaceutical Journal of Chinese People's Liberation Army 2008;24(3):268-269.

[11] Carbajo-Lozoya J, Muller MA, Kallies S, Thiel V, Drosten C, von Brunn A. Replication of human coronaviruses SARS-CoV, HCoVNL63 and HCoV-229E is inhibited by the drug FK560. Virus Res 2012;165(1):112-117. doi:10.1016/j.virusres.2012.02.002.

[12] Chan JF, Chan KH, Kao RY, To KK, Zheng BJ, Li CP, et al. Broad-spectrum antivirals for the emerging Middle East respiratory syndrome coronavirus. J Infect 2013;67(6):606-616. doi:10.1016/j.jinf.2013.09.029.

[13] Zhu YP, Wang P, Xia BR, Tang YT, Wang Q. Screening and inhibition kinetics of SARS coronavirus major protease inhibitors (in Chinese). Chin J Biotech 2016;36(4):35-42. doi:10.13523/j.cb.20160406.

[14] lijima S. Helical microtubules of graphitic carbon. Nature 1991;354:56-58. doi:10.1038/354056a0.

[15] Baker SE, Cai W, Lasseter TL, Weidkamp KP, Hamers RJ. Covalently bonded adducts of deoxyribonucleic acid (DNA) oligonucleotides with single-wall carbon nanotubes: synthesis and hybridization. Nano Lett 2002;2:1413-1417. doi:10.1021/nl025729f.

[16] Besteman K, Lee JO, Wiertz FGM, Heering HA, Dekker C. Enzymecoated carbon nanotubes as single-molecule biosensors. Nano Lett 2003;3:727-730. doi:10.1021/nl034139u.

[17] Maestro LM, Haro-Gonzalez P, del Rosal B, Ramiro J, Caamaño AJ, Carrasco $E$, et al. Heating efficiency of multi-walled carbon nanotubes in the first and second biological windows. Nanoscale 2013;5(17):7882-7889. doi:10.1039/c3nr01398g.

[18] Burke A, Ding X, Singh R, Kraft RA, Levi-Polyachenko N, Rylander MN, et al. Long-term survival following a single treatment of kidney tumors with multiwalled carbon nanotubes and near-infrared radiation. PNAS 2009;106(31):12897-12902. doi:10.1073/pnas.0905195106.

[19] Bi S, Su XJ, Hou GL, Liu CH, Xiao Z, Xiao YD, et al. Investigations on oxidation cutting and dispersibility of multi-walled carbon nanotubes. New Carbon Mater 2014;29(2):109-117. doi:10.1016/j.carbon.2014.04.040.

[20] Ma HY, Tong LF, Xu ZB, Fang ZP. Functionalizing carbon nanotubes by grafting on intumescent flame retardant: nanocomposite synthesis, morphology, rheology, and flammability. Adv Funct Mater 2008;18(3):414-421. doi:10.1002/adfm.200700677.

[21] Walls AC, Park YJ, Tortorici MA, Wall A, McGuire AT, Veesler D. Structure, function, and antigenicity of the SARS-CoV-2 spike glycoprotein. Cell 2020;181(2):281-292.e6. doi:10.1016/j.cell.2020.02.058.

[22] Zhao Y, Zhao ZX, Wang YJ, Zhou YQ, Ma Y, Zuo W. Single-cell RNA expression profiling of ACE2, the receptor of SARS-CoV-2. bioRxiv 2020:919985. doi:10.1101/2020.01.26.919985.

[23] Ferron F, Subissi L, Silveira De Morais AT, Le NTT, Sevajol M, Gluais L, et al. Structural and molecular basis of mismatch correction and ribavirin excision from coronavirus RNA. PNAS 2018;115(2):E162-E171. doi:10.1073/pnas.1718806115. 